\title{
Antimicrobial activity of binary combinations of natural and synthetic phenolic antioxidants against Enterococcus faecalis
}

\author{
J. Gutiérrez-Fernández, ${ }^{* 1}$ M. R. García-Armesto, $\dagger^{2}$ R. Álvarez-Alonso, ${ }^{*}$ P. del Valle, ${ }^{*} D$. de Arriaga, ${ }^{*}$ \\ and J. Rúa*2,3 \\ *Department of Molecular Biology, and \\ †Department of Food Hygiene and Food Technology, University of León, 24071 León, Spain
}

\begin{abstract}
This study investigated the antimicrobial activity of 3 natural (thymol, carvacrol, and gallic acid) and 2 synthetic [butylated hydroxyanisole (BHA) and octyl gallate] phenolic compounds, individually and in binary combinations, on 4 dairy isolates of Enterococcus faecalis with different virulence factors ( $\beta$-hemolytic, gelatinase, or trypsin activities; acquired resistance to erythromycin or tetracycline; and natural resistance to gentamicin). A checkerboard technique and a microdilution standardized method were used. All compounds individually tested exhibited antimicrobial activity against $E$. faecalis, with minimal inhibitory concentrations (MIC) ranging from $30 \mu \mathrm{g} / \mathrm{mL}$ (octyl gallate) to $3,150 \mu \mathrm{g} / \mathrm{mL}$ (gallic acid), although no significant differences were detected among strains to each phenolic compound. Carvacrol in combination with thymol or gallic acid, and gallic acid combined with octyl gallate showed partial synergistic inhibition of all E. faecalis strains. The most effective combinations were thymol + carvacrol and gallic acid + octyl gallate, as the MIC for each of these compounds was reduced by 67 to $75 \%$ compared with their respective individual MIC. These results highlight the possibility of using combinations of these phenolic compounds to inhibit the growth of potential virulent or spoilage E. faecalis strains by reducing the total amount of additives used in dairy foods.
\end{abstract}

Key words: phenolic compound, antimicrobial activity, synergistic effect, Enterococcus faecalis

\footnotetext{
Received January 30, 2013.

Accepted April 24, 2013.

${ }^{1}$ Current address: Department of Crystallography and Structural Biology, Instituto de Química-Física Rocasolano, CSIC, Serrano 119, 28006 Madrid, Spain.

${ }^{2}$ María Rosario García-Armesto and Javier Rúa contributed with equal responsibility to the conduct of this study.

${ }^{3}$ Corresponding author: javier.rua@unileon.es
}

\section{INTRODUCTION}

Enterococci are commensal bacteria of the gastrointestinal tract of humans and other mammals, as well as common members of the bacterial communities of food fermentations (EFSA, 2011). These microorganisms can act as opportunistic agents, causing a variety of infections in humans; however, the pathogenesis of enterococcal infections is still poorly understood (Tendolkar et al., 2003). Several potential virulence factors have been identified in enterococcal isolates and are thought to play a role in the pathogenesis of enterococcal infections; these include, among others, the secreted toxin cytolysin/hemolysin and the secreted proteases with gelatinase and trypsin activities (Pillar and Gilmore, 2004). Other mechanisms by which the enterococci can deviate from their commensal behavior in mammals is through the acquisition of new traits that allow the bacterium to overcome host defenses and colonize new niches. In this context, acquired antimicrobial resistance is considered one of the many traits that virulent enterococci possess compared with commensal isolates (Shankar et al., 2002). Trends to increase the prevalence of these organisms as nosocomial pathogens have been frequently observed (Malani et al., 2002). In particular, Enterococcus faecalis is usually the enterococcal species most frequently isolated from human clinical specimens, representing 80 to $90 \%$ of the isolates, followed by Enterococcus faecium (Martins et al., 2011). Antibiotic resistance is common in some dairy food enterococcal isolates (Jamet et al., 2012), and several studies confirm widespread incidence of erythromycin-, kanamycin-, and tetracycline-resistant enterococci in foods (for review, see Ogier and Serror, 2008).

Phenolic compounds are defined as substances that possess an aromatic ring bearing one or more hydroxyl substituents, including functional derivatives (e.g., acids, esters, methyl esters, glycosides; Shahidi and Ho, 2005). These compounds are considered to have potential human health beneficial effects (antioxidant, anticarcinogenic, and antiinflammatory activities; Garrote et al., 2004) and they are antioxidants or flavoring agents in food (Rúa et al., 2010). Additionally, phenolic 
compounds have been shown to possess antimicrobial activity against a wide range of microorganisms (Davidson and Taylor, 2007), a property that can be exploited to inhibit growth of foodborne bacteria and to extend the shelf life of processed food.

Previous studies in our laboratory on the antimicrobial efficacy of 20 natural and synthetic phenolic compounds against several gram-positive and gramnegative microorganisms revealed that among the natural ones, gallic acid, carvacrol, and thymol were the most effective and the most uniform in their antimicrobial effect; of the synthetic compounds studied, octyl gallate was the most effective and butylated hydroxyanisole (BHA) the most uniform in antimicrobial activity (Rúa et al., 2010, 2011; Gutiérrez-Larraínzar et al., 2012, 2013; Figure 1). Additionally, both synthetic compounds mentioned are used frequently by the food industry as antioxidants.

Little work has been published concerning the influence of combinatory effect of phenolic compounds on growth of foodborne pathogenic or spoilage microorganisms, and particularly on E. faecalis. These combinations could exhibit synergistic antimicrobial properties, wherein one or more components of the combination assayed could be used at a lower concentration than that effective when used individually. This would reduce the total amount of additives, particularly synthetic ones, to processed foods, improving their quality and safety and acceptance by consumers, who have become increasingly critical of the use of chemical preservatives in foods.

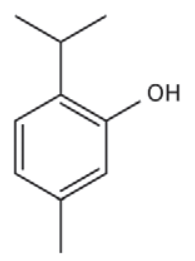

Thymol<smiles>COc1ccc(O)c(C(C)(C)C)c1</smiles>

$\mathrm{BHA}$

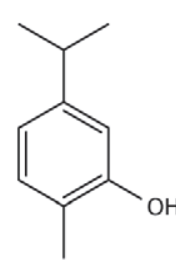

Carvacrol

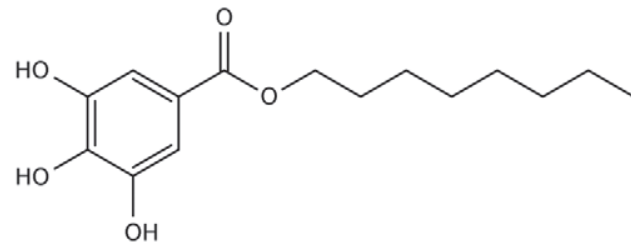

Octyl gallate
Figure 1. Chemical structures of the phenolic compounds used in this study.
In this work, we selected several E. faecalis strains isolated from sheep dairy products, which showed different potential virulence factors ( $\beta$-hemolytic, gelatinase, or trypsin activities) or phenotypic resistance to some antibiotics that are considered by World Health Organization as "critically (gentamicin and erythromycin) or highly (tetracycline) important antimicrobials" (EFSA, 2008), because of their use in human and veterinary medicine. The aim of this study was to investigate the antimicrobial efficacy of 3 natural (thymol, carvacrol, and gallic acid) and 2 synthetic (BHA and octyl gallate) phenolic compounds in binary combinations against $E$. faecalis strains with different virulence traits or resistance to antibiotics, to identify a synergistic combination that could be effective against these strains, reducing the total amount of phenolic compounds necessary to control these microorganisms in standard culture medium.

\section{MATERIALS AND METHODS}

\section{Cultures and Microorganisms}

Four strains of E. faecalis isolated from raw ewe milk and blended (ewe and cow) cheese, were used in this study. The strains were previously characterized taxonomically (by classical and molecular methods), biochemically, technologically, and physiologically (Monteagudo-Mera et al., 2011, 2012). Virulence traits and antibiotics against which the strains showed some resistance are summarized in Table 1 . The isolates were supplied by García Armesto (Department of Food Hygiene and Food Technology, University of León, León, Spain).

These strains were grown aerobically in trypticasein soy agar (Laboratorios Conda, Madrid, Spain) plates for $24 \mathrm{~h}$ at $30^{\circ} \mathrm{C}$. Then, isolated colonies were grown in $4 \mathrm{~mL}$ of tryptic soy broth (Oxoid Ltd., Basingstoke, UK) plus 1\% (wt/vol) yeast extract (Oxoid Ltd.) for 24 $\mathrm{h}$ at $30^{\circ} \mathrm{C}$. These cultures were maintained in Eppendorf tubes in the presence of $20 \%$ ( $\mathrm{vol} / \mathrm{vol}$ ) glycerol at $-80^{\circ} \mathrm{C}$. Frozen stock cultures were activated by transferring $20 \mu \mathrm{L}$ into $4 \mathrm{~mL}$ of tryptic soy broth $+1 \%$ yeast extract and incubating for $24 \mathrm{~h}$ at $30^{\circ} \mathrm{C} \pm 1{ }^{\circ} \mathrm{C}$.

\section{Chemicals and Preparation of Stock Solutions}

Thymol, carvacrol, and BHA were all obtained from Sigma-Aldrich Co. (St. Louis, MO). Octyl gallate was obtained from Cymit Química (Barcelona, Spain), and gallic acid was obtained from Fluka and Riedel-deHäen (Buchs SG, Switzerland).

Stock solutions of gallic acid (at a final concentration of $12.8 \mathrm{mg} / \mathrm{mL}$ ) and thymol, carvacrol, BHA, and octyl 


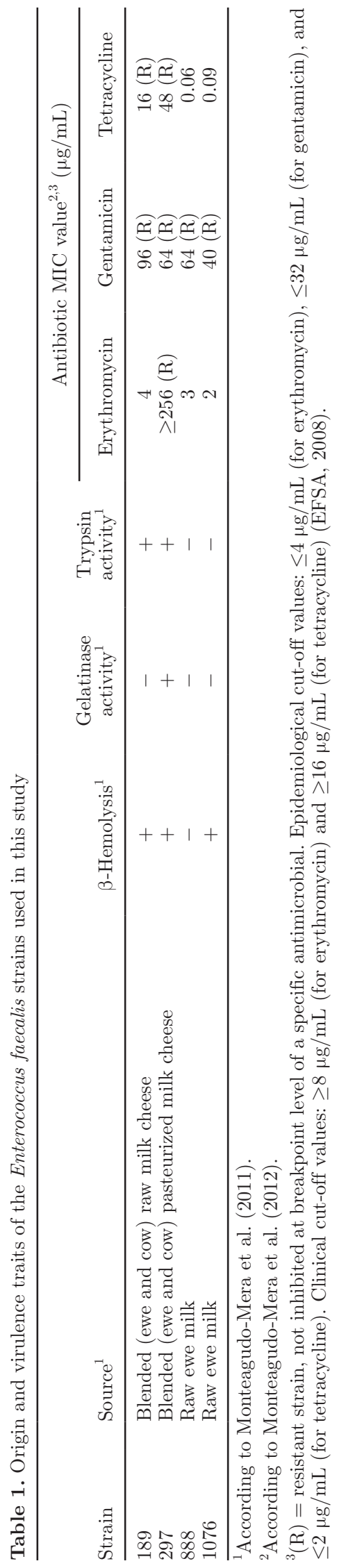

gallate (at a final concentration of $3.2 \mathrm{mg} / \mathrm{mL}$ ) were freshly prepared by dissolving the appropriate amount of the phenolic compound in 1 part of $95 \%$ ( $\mathrm{vol} / \mathrm{vol}$ ) ethanol and 9 parts of Mueller-Hinton broth (MHB). The compounds were first dissolved in the alcohol by constant shaking before adding the MHB. The stock solutions were protected from exposure to light. The antimicrobial activity of 5 to $10 \%$ ( $\mathrm{vol} / \mathrm{vol}$ ) ethanol was studied on a separate microtiter plate before the checkerboard assay.

\section{MIC Determination and Synergy Testing}

Determinations of MIC and synergy testing were performed by the checkerboard method in microtiter plates with MHB. The method to carry out the antimicrobial microdilution assay to estimate the MIC values of the selected phenolic compounds was that described in ISO Standard 20776-1 (ISO, 2006). Briefly, after checking the recovery ability and purity of strains, inocula for the antimicrobial assays were prepared by diluting the overnight cultures with sterile MHB to obtain a concentration of $5 \times 10^{5} \mathrm{cfu} / \mathrm{mL}$. The concentration of microorganisms was checked by the Miles and Misra technique (ICMSF, 1978).

Synergy tests were performed in a 96-well microtiter plate containing 2 antimicrobial agents in 2-fold dilutions dispensed in a checkerboard fashion on the day of the assay. Different concentrations in serial double dilutions were prepared from the stock solution in sterile glass tubes. Twenty-five microliters of each solution of one compound was added to the rows (12 wells in total) in diminishing concentrations, by using a 12-channel micropipette, and $25 \mu \mathrm{L}$ of the other compound was added to the columns in diminishing concentrations ( 8 wells in total). A second plate for each combination and strain was prepared, dephasing the 2 -fold serial dilutions of each compound to obtain intermediate values and increasing the experimental data for the same interval (Horrevorts et al., 1987). After that, aliquots of $50 \mu \mathrm{L}$ of standardized bacterial inoculum (at a concentration of $5 \times 10^{5} \mathrm{cfu} / \mathrm{mL}$ ) were added to each well, giving a final volume of $100 \mu \mathrm{L}$. Controls containing MHB and inoculum alone, and antimicrobial compounds separately with the inoculum were used in the same plate. The microplates were sealed using a sterile microporous film (Sigma-Aldrich Co.), mixed manually, and incubated for $24 \mathrm{~h}$ at $37^{\circ} \mathrm{C}$. Then, the growth in each well was quantified by using a visual observation method or a spectrophotometric method, in which optical density was read at $620 \mathrm{~nm}$ on a microplate reader (Anthos 2020, Biochrom Ltd., Cambridge, UK). At least 2 trials on different days were carried out in duplicate for each strain and binary combination. 
The MIC of each compound alone or in combination was defined as the minimal concentration of antimicrobial compound that inhibited visible growth of the strain tested (Barry, 1976). Individual MIC of each compound were estimated in the same microtiter plate from the data of the column or the row in which one of the compounds was absent. The MIC data were transformed to fractional inhibitory concentrations (FIC). The FIC of an individual antimicrobial compound is the ratio of the MIC of the antimicrobial in combination with a second compound to the MIC of the antimicrobial by itself, as follows:

$$
\mathrm{FIC}_{\mathrm{A}}=(\mathrm{MIC} \text { of } \mathrm{A} \text { with } \mathrm{B}) / \mathrm{MIC} \text { of } \mathrm{A} .
$$

The FIC index (FICI) was calculated as follows from the FIC of the individual antimicrobials:

$$
\mathrm{FICI}=\mathrm{FIC}_{\mathrm{A}}+\mathrm{FIC}_{\mathrm{B}} .
$$

For the FICI calculation, we used the wells nearest to $1 / 2 \mathrm{MIC}_{\mathrm{A}}, 1 / 2 \mathrm{MIC}_{\mathrm{B}}$, as they tend to be the most informative (Hsieh et al., 1993). The criteria used to determine the type of combined antimicrobial effect were as follows: FICI $<0.5$ indicates synergy; FICI $\geq 0.5$ and $<1.0$ indicates partial synergy; FICI $=1.0$ indicates additive effect; FICI $\geq 2.0$ indicates antagonism (Sato et al., 2004). The interaction of antimicrobial combinations was determined with the isobologram previously described by Davidson and Parish (1989).

\section{Statistical Analysis}

All microbiological data were expressed as means of at least quadruplicate measurements. For individual MIC, one-way nonparametric analysis was carried out to test any differences among individual strains in the same bacterial species (intraspecific differences) and among individual bacterial species (interspecific differences). Statistical analysis was performed by using the program R for Windows, developed by $\mathrm{R}$ Development Core Team (2009). Significant differences $(P<0.05)$ between means were determined by Kruskal-Wallis (in- traspecific differences) and Mann-Whitney-Wilcoxon methods (interspecific differences).

\section{RESULTS}

\section{MIC of the Isolated Compounds}

The MIC values of 3 natural phenols (thymol, carvacrol, and gallic acid) and 2 synthetic phenols (BHA and octyl gallate) are summarized in Table 2. The individual MIC for each compound was calculated from the corresponding binary combinations performed in the study in the absence of one of the compounds. The behavior was similar for all strains and one inhibitor. However, we found statistical differences among the 5 compounds used (Figure 2). Octyl gallate was the strongest inhibitor for all strains of E. faecalis used in this study, whereas gallic acid was the weakest inhibitor, with MIC values 100 times higher than that of its alkyl derivative. Butylated hydroxyanisole, the other synthetic compound assayed, was a better inhibitor than the 3 natural phenols used. The antibacterial order of the natural phenolic compound was carvacrol $>$ thymol > gallic acid.

\section{Combination Effects of Natural Phenolic Compounds}

When carvacrol was combined with thymol or gallic acid, synergistic effects on growth was observed for the 4 strains of $E$. faecalis, in which the FICI ranged from 0.5 to 0.75 (partial synergy; Table 3). However, the combination of gallic acid and thymol showed additive activity against strains 888 and 1076 (FICI of 1.0). The levels of carvacrol or thymol required for inhibition in the presence of the other one ranged from $1 / 4$ to $1 / 2$ versus the corresponding natural phenolic compound alone $(200$ and $400 \mu \mathrm{g} / \mathrm{mL}$ for carvacrol, 400 and 600 $\mu \mathrm{g} / \mathrm{mL}$ for thymol). Meanwhile, for gallic acid, levels needed for inhibition ranged from $1 / 8$ to $1 / 2$ versus the MIC value of the compound alone (400 to 1,200 $\mu \mathrm{g} / \mathrm{mL}$ ). As an example of partial synergy of natural phenolic compounds, Figure 3A shows the mode of interaction of thymol and carvacrol as a FIC isobologram against E. faecalis strain 189. The degree of synergy

Table 2. Minimum inhibitory concentrations $(\mu \mathrm{g} / \mathrm{mL})$ of natural and synthetic phenolic compounds against 4 strains of Enterococcus faecalis ${ }^{1}$

\begin{tabular}{lccccc}
\hline Strain & Thymol & Carvacrol & Gallic acid & BHA $^{2}$ & $\begin{array}{c}\text { Octyl } \\
\text { gallate }\end{array}$ \\
\hline 189 & $1,200 \pm 300$ & $700 \pm 100$ & $3,300 \pm 200$ & $300 \pm 0$ & $30 \pm 7$ \\
297 & $1,100 \pm 400$ & $700 \pm 100$ & $3,300 \pm 200$ & $300 \pm 0$ & $30 \pm 7$ \\
888 & $1,000 \pm 400$ & $700 \pm 100$ & $2,900 \pm 600$ & $300 \pm 0$ & $30 \pm 7$ \\
1076 & $1,100 \pm 400$ & $750 \pm 100$ & $3,100 \pm 500$ & $300 \pm 0$ & $30 \pm 7$ \\
\hline
\end{tabular}

${ }^{1}$ Values are the means of at least 4 experiments in duplicate \pm SEM. Within a column (antimicrobial compound), values were not significantly different $(P>0.05)$.

${ }^{2}$ Butylated hydroxyanisole. 


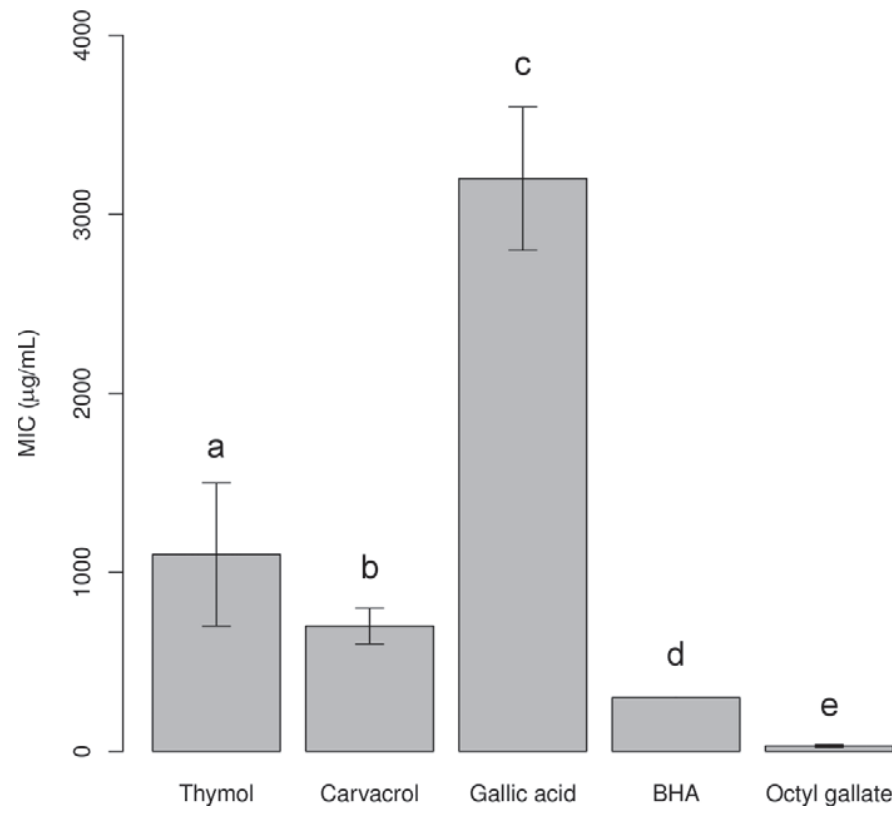

Figure 2. Minimum inhibitory concentrations of phenolic compounds against the 4 Enterococcus faecalis strains studied. Different letters (a, b, c, d, e) indicate significant differences $(P<0.05)$ among antimicrobial compounds. BHA = butylated hydroxyanisole.

observed is indicated by the curve of the line away from the theoretical additive (dotted) line toward the intersection of the $x$ - and $y$-axes.

\section{Combination Effects of Natural and Synthetic Phenolic Compounds}

The influence of the synthetic phenolic compounds (BHA or octyl gallate) on the inhibition of thymol, carvacrol, and gallic acid against the 4 strains of E. faecalis is summarized in Table 4. In general, the synergistic effect was less than that observed in the combinations of the 3 natural phenols, as we obtained 6 additive effects in the combination with BHA and 4 in the combination with octyl gallate. Also, the pattern of behavior of the strains for these combinations was different. Particularly for the combination of BHA with thymol or carvacrol, strain 888 was the only one for which we detected a partial synergy, but we could not obtain a result for this strain in the combination of $\mathrm{BHA}$ and gallic acid, as we were not able to estimate the FIC values by following the method used in this study. In contrast, for strain 1076, we obtained an additive effect of BHA both with thymol and with gallic acid. It is also interesting that for the combinations of octyl gallate with the 3 natural phenols, we detected an additive effect for 2 different strains in the combination with thymol (strains 888 and 1076) or carvacrol (189 and 297), but a partial synergy was estimated for all strains in the combination of gallic acid with this alkyl gallate.

The levels of the synthetic phenols required for inhibition in the presence of the natural phenols diminished as follows: for BHA, they ranged from 1/2 compared with the corresponding MIC value alone in the presence of carvacrol; $1 / 4$ in the presence of gallic acid and from $1 / 4$ to $<1 / 2$ in presence of thymol; for octyl gallate, the MIC in combination were reduced $1 / 4,1 / 2$, and $<1 / 2$, compared with the corresponding MIC alone in the presence of gallic acid, thymol, and carvacrol, respectively.

With regard to the natural phenols, the presence of BHA reduced the levels of gallic acid to $<1 / 2$ for the 3 assayed strains, indicating higher intraspecific variety in the reduction of the levels of thymol (from $1 / 3$ to $<1 / 2$ ) and carvacrol (from $1 / 8$ to $1 / 2$, with strain 888 being the most sensitive, as its growth was inhibited with a lower concentration of carvacrol in combination with BHA). On the other hand, the presence of octyl

Table 3. Antimicrobial activities of natural phenolic compounds in binary combinations (comb) against 4 strains of Enterococcus faecalis ${ }^{1,2}$

\begin{tabular}{|c|c|c|c|c|c|c|c|}
\hline Combination & Strain & $\begin{array}{c}\mathrm{MIC}_{\mathrm{A}} \\
\text { (alone/comb) }\end{array}$ & $\mathrm{FIC}_{\mathrm{A}}$ & $\begin{array}{c}\mathrm{MIC}_{\mathrm{B}} \\
\text { (alone/comb) }\end{array}$ & $\mathrm{FIC}_{\mathrm{B}}$ & FICI & $\begin{array}{l}\text { Interpretation } \\
\text { of } \mathrm{FICI}^{3}\end{array}$ \\
\hline \multirow[t]{4}{*}{ Thymol + carvacrol } & 189 & $1,600 / 400$ & 0.25 & $800 / 200$ & 0.25 & 0.50 & Partial synergy \\
\hline & 297 & $1,600 / 400$ & 0.25 & $800 / 200$ & 0.25 & 0.50 & Partial synergy \\
\hline & 888 & $1,600 / 400$ & 0.25 & $800 / 200$ & 0.25 & 0.50 & Partial synergy \\
\hline & 1076 & $1,600 / 400$ & 0.25 & $800 / 200$ & 0.25 & 0.50 & Partial synergy \\
\hline \multirow[t]{4}{*}{ Gallic acid + thymol } & 189 & $3,200 / 800$ & 0.25 & $1,200 / 800$ & 0.67 & 0.92 & Partial synergy \\
\hline & 297 & $3,200 / 1,200$ & 0.38 & $1,200 / 600$ & 0.50 & 0.88 & Partial synergy \\
\hline & 888 & $2,400 / 1,200$ & 0.50 & $1,200 / 600$ & 0.50 & 1.00 & Additive \\
\hline & 1076 & $2,400 / 1,200$ & 0.50 & $1,200 / 600$ & 0.50 & 1.00 & Additive \\
\hline \multirow[t]{4}{*}{ Gallic acid + carvacrol } & 189 & $3,200 / 800$ & 0.25 & $800 / 400$ & 0.50 & 0.75 & Partial synergy \\
\hline & 297 & $3,200 / 800$ & 0.25 & $800 / 400$ & 0.50 & 0.75 & Partial synergy \\
\hline & 888 & $3,200 / 400$ & 0.12 & $800 / 400$ & 0.50 & 0.62 & Partial synergy \\
\hline & 1076 & $3,200 / 800$ & 0.25 & $800 / 400$ & 0.50 & 0.75 & Partial synergy \\
\hline
\end{tabular}

${ }^{1}$ Values of the MIC are the mean of at least 2 duplicate experiments. $\mathrm{MIC}_{\mathrm{A}}$ and $\mathrm{MIC}_{\mathrm{B}}$ are the MIC of 2 antimicrobial compounds used alone or in combination.

${ }^{2} \mathrm{FIC}=$ fractional inhibitory concentration; $\mathrm{FIC}_{\mathrm{A}}$ and $\mathrm{FIC}_{\mathrm{B}}$ are the $\mathrm{FIC}$ for compounds $\mathrm{A}$ and $\mathrm{B}$, respectively; $\mathrm{FICI}=$ fractional inhibitory concentration index.

${ }^{3}$ According to Sato et al. (2004). 

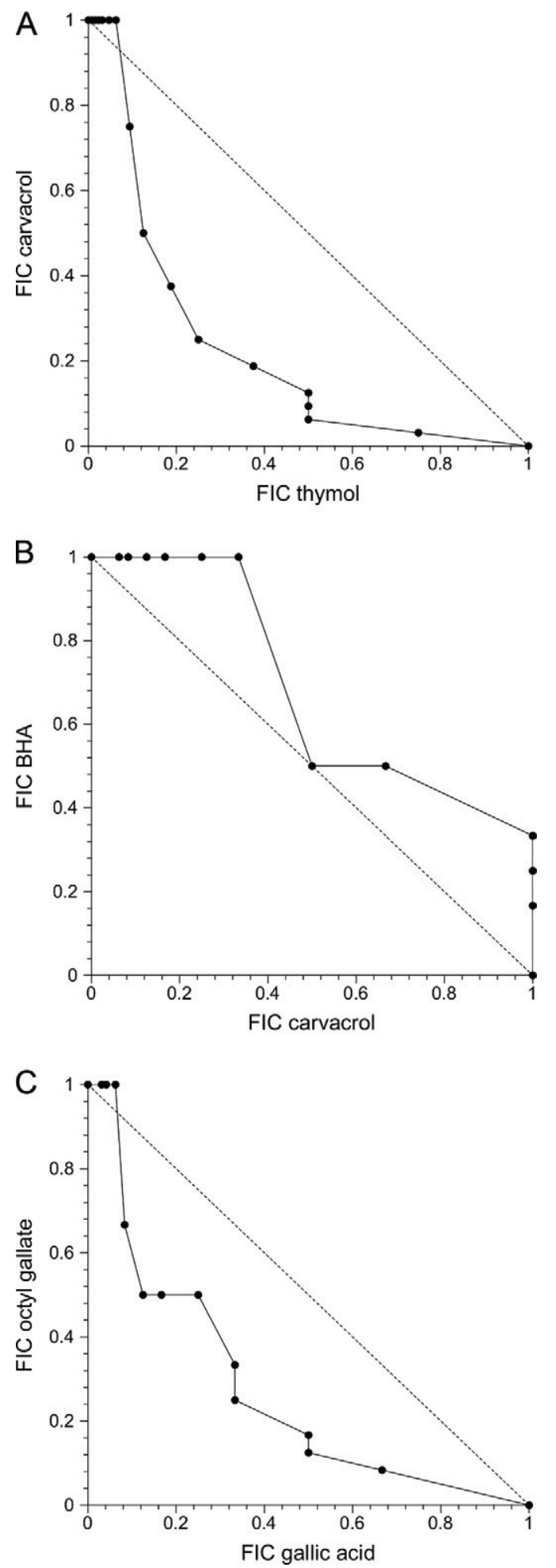

Figure 3. Fractional inhibitory concentration (FIC) isobolograms for combinations of thymol and carvacrol (A), carvacrol and butylated hydroxyanisole (BHA; B), or gallic acid and octyl gallate (C) against Enterococcus faecalis. The dotted line indicates the theoretical additive line. gallate diminished the MIC of gallic acid to one-third for the 4 strains; diminished the MIC levels of carvacrol to one-half or one-quarter; and diminished the MIC levels of thymol to one-third or one-half compared with MIC of gallic acid, carvacrol, or thymol alone, respectively. Strain 888 was again the most sensitive to the combination of carvacrol and octyl gallate. The modes of interaction of BHA with carvacrol and octyl gallate with gallic acid for strain 189 are presented as FIC isobolograms in Figures $3 \mathrm{~B}$ and $3 \mathrm{C}$. An additive effect or synergism was observed for these combinations, respectively.

\section{DISCUSSION}

Resistance to several commonly used antimicrobial agents is a remarkable characteristic of most enterococcal species, particularly E. faecalis and E. faecium, the enterococci most frequently associated with human infections. In this study, the antimicrobial effectiveness of natural phenolic compounds was lesser for the 4 strains of $E$. faecalis than that reported in previous studies for other gram-positive genera of bacteria. Hence, the mean MIC value for thymol $(1,100 \mu \mathrm{g} / \mathrm{mL})$ was about twice as high as the mean MIC values for this compound against Staphylococcus aureus $(400 \mu \mathrm{g} / \mathrm{mL})$ and against Bacillus cereus $(580$ g/mL; Rúa et al., 2011; Gutiérrez-Larraínzar et al., 2012). With regard to carvacrol, our mean MIC value against 4 strains of E. faecalis was $712.5 \mu \mathrm{g} / \mathrm{mL}$, about twice as high as that reported by us in a previous study against Staph. aureus $(367 \mu \mathrm{g} / \mathrm{mL})$ and B. cereus (330 $\mu \mathrm{g} / \mathrm{mL}$; Gutiérrez-Larraínzar et al., 2012). The mode of action of thymol and carvacrol has received much attention from researchers (for review, see Hyldgaard et al., 2012). Both phenolic monoterpenoids are structurally very similar, having the hydroxyl group at a different position on the phenolic ring (Figure 1). The primary mode of antibacterial action of thymol is not fully known but is expected to be similar to that of carvacrol, causing structural and functional damage to the cytoplasmic membrane (Sikkema et al., 1995). The hydroxyl group combined with a system of delocalized electrons confers an acidic character to these molecules, which could allow them to release protons inside the cells, causing cytoplasmic acidification. It has been proposed for carvacrol that the hydroxyl group could function in membrane transport, carrying $\mathrm{H}^{+}$into the cell cytoplasm and transporting $\mathrm{K}^{+}$out; the maintenance of internal $\mathrm{pH}$ homeostasis implies the existence of an electrochemical proton gradient generated by an $\mathrm{H}^{+}$-ATPase plasma membrane, which could cause a decrease in the concentration of ATP and lead to cell death (Ultee et al., 2002; Ben Arfa et al., 2006). This similar antimicrobial mechanism of thymol and 
Table 4. Antimicrobial activities of butylated hydroxyanisole (BHA) or octyl gallate with natural phenolic compounds in binary combinations (comb) against 4 strains of Enterococcus faecalis ${ }^{1,2}$

\begin{tabular}{|c|c|c|c|c|c|c|c|}
\hline Combination & Strain & $\begin{array}{c}\mathrm{MIC}_{\mathrm{A}} \\
\text { (alone/comb) }\end{array}$ & $\mathrm{FIC}_{\mathrm{A}}$ & $\begin{array}{c}\mathrm{MIC}_{\mathrm{B}} \\
\text { (alone/comb) }\end{array}$ & $\mathrm{FIC}_{\mathrm{B}}$ & FICI & $\begin{array}{l}\text { Interpretation } \\
\text { of } \text { FICI }^{3}\end{array}$ \\
\hline \multirow[t]{2}{*}{$\mathrm{BHA}+$ thymol } & 189 & $300 / 75$ & 0.25 & $1,200 / 600$ & 0.50 & 0.75 & Partial synergy \\
\hline & 1076 & $300 / 200$ & 0.66 & $1,200 / 400$ & 0.33 & 1.00 & Additive \\
\hline \multirow[t]{2}{*}{ BHA + carvacrol } & 189 & $300 / 150$ & 0.50 & $600 / 300$ & 0.50 & 1.00 & Additive \\
\hline & 297 & $300 / 150$ & 0.50 & $600 / 150$ & 0.25 & 0.75 & Partial synergy \\
\hline \multirow[t]{4}{*}{$\mathrm{BHA}+$ gallic acid } & 189 & $300 / 75$ & 0.25 & $3,200 / 2,400$ & 0.75 & 1.00 & Additive \\
\hline & 297 & $300 / 75$ & 0.25 & $3,200 / 2,400$ & 0.75 & 1.00 & Additive \\
\hline & 888 & $300 / \mathrm{ND}$ & ND & $2,400 / \mathrm{ND}$ & ND & ND & ND \\
\hline & 1076 & $300 / 75$ & 0.25 & $3,200 / 2,400$ & 0.75 & 1.00 & Additive \\
\hline \multirow[t]{3}{*}{ Octyl gallate + thymol } & 189 & $25 / 12.5$ & 0.50 & $900 / 300$ & 0.33 & 0.83 & Partial synergy \\
\hline & 297 & $25 / 12.5$ & 0.50 & $900 / 300$ & 0.33 & 0.83 & Partial synergy \\
\hline & 888 & $25 / 12.5$ & 0.50 & $600 / 300$ & 0.50 & 1.00 & Additive \\
\hline \multirow[t]{4}{*}{ Octyl gallate + gallic acid } & 189 & $37.5 / 9.37$ & 0.25 & $3,600 / 1,200$ & 0.33 & 0.58 & Partial synergy \\
\hline & 297 & $37.5 / 9.37$ & 0.25 & $3,600 / 1,200$ & 0.33 & 0.58 & Partial synergy \\
\hline & 888 & $37.5 / 9.37$ & 0.25 & $3,600 / 1,200$ & 0.33 & 0.58 & Partial synergy \\
\hline & 1076 & $37.5 / 9.37$ & 0.25 & $3,600 / 1,200$ & 0.33 & 0.58 & Partial synergy \\
\hline
\end{tabular}

${ }^{1}$ Values of the MIC are the mean of at least 2 duplicate experiments. $\mathrm{MIC}_{\mathrm{A}}$ and $\mathrm{MIC}_{\mathrm{B}}$ are the MIC of 2 antimicrobial compounds used alone or in combination.

${ }^{2} \mathrm{FIC}=$ fractional inhibitory concentration; $\mathrm{FIC}_{\mathrm{A}}$ and $\mathrm{FIC}_{\mathrm{B}}$ are the $\mathrm{FIC}$ for compounds $\mathrm{A}$ and $\mathrm{B}$, respectively; FICI $=$ fractional inhibitory concentration index.

${ }^{3}$ According to Sato et al. (2004).

carvacrol could support the MIC for these compounds being closer than those for the other 3 phenolic compounds studied (Table 1).

Gallic acid was less effective than thymol and carvacrol (and its alkyl derivative octyl gallate). This could be expected because the $\mathrm{pH}$ (6.5) of the culture medium (MHB) is higher than the $\mathrm{pK}_{a}$ value (acid dissociation constant) of the carboxyl group of the phenolic acids (approximately 4.5). As a result of this, at the $\mathrm{pH}$ of the medium, these acids are mostly dissociated, and the most antimicrobial effective form of an acid is the undissociated form (Davidson and Taylor, 2007) because it is more soluble in the cytoplasmic membrane (Ramos-Nino et al., 1996). Compared with previously published studies, our mean MIC values for gallic acid against the 4 strains of $E$. faecalis $(3,150 \mu \mathrm{g} / \mathrm{mL})$ were higher than that reported against Staph. aureus (560 $\mu \mathrm{g} / \mathrm{mL}$; Gutiérrez-Larraínzar et al., 2012), but similar to those reported previously when using a single strain of Staph. aureus: 3,200 $\mu \mathrm{g} / \mathrm{mL}$ (Kubo et al., 2003), and 2 strains of lactic acid bacteria: Lactobacillus plantarum $(3,740 \mu \mathrm{g} / \mathrm{mL})$ and Lactobacillus hamnesii $(4,560 \mu \mathrm{g} /$ mL; Sánchez-Maldonado et al., 2011). One possible explanation for the resistance of lactic acid bacteria to phenolic compounds is that, in keeping with prior observations, lactobacilli metabolize phenolic acids by strain-specific decarboxylation or reduction of these compounds (Svensson et al., 2010).

Butylated hydroxyanisole is a simple phenol approved for use in foods as an additive in the European Union (E 320, Commission Regulation (EU) No. 1129/2011) and United States (US Food and Drug Administration/ Center for Food Safety and Applied Nutrition; EAFUS (Everything Added to Food in the United States), 2011). This compound is usually used as antioxidant as it is a synthetic analog of vitamin $\mathrm{E}$; nevertheless, the antimicrobial activity of this and other synthetic phenolics have been recognized in some previous studies (reviewed by López-Malo et al., 2005). According to our results, the mean MIC value of BHA against E. faecalis was $300 \mu \mathrm{g} /$ $\mathrm{mL}$, which is in the range of the MIC values previously reported against Staph. aureus, about 200 to $300 \mu \mathrm{g} /$ mL (Gailani and Fung, 1984; Gutiérrez-Larraínzar et al., 2013), although it is lower than the MIC values reported against B. cereus by Gutiérrez-Larraínzar et al. (2013; $1,228 \mu \mathrm{g} / \mathrm{mL}$ ) and Griffiths (2010; from 1,000 to 5,000 $\mu \mathrm{g} / \mathrm{mL}$ ). Different methodologies, together with specific features of the gram-positive genera, could explain the differences obtained.

Octyl gallate is an alkyl derivative of gallic acid (3,4,5-trihydroxybenzoic acid) mainly used as antioxidant and approved for use in foods as additive in the 
European Union (E 311) and United States (EAFUS, 2011). In the present study, we found that the mean MIC value of octyl gallate against E. faecalis $(30 \mu \mathrm{g} /$ $\mathrm{mL}$ ) was similar to that previously reported against Staph. aureus $(18 \mu \mathrm{g} / \mathrm{mL})$ and against sensitive strains of B. cereus (49 $\mu \mathrm{g} / \mathrm{mL}$; Gutiérrez-Larraínzar et al., 2013). If we compare the results obtained in this study for octyl gallate and gallic acid against E. faecalis, we can see that the mean MIC values for the phenolic acid is higher (2 orders of magnitude) than that obtained for the $\mathrm{C}_{8}$ ester (octyl gallate). This agrees with the hypothesis that the antibacterial activity of alkyl gallates against Bacillus (particularly Bacillus subtilis) is a parabolic function of their lipophilicity and is maximized with alkyl chain length between $\mathrm{C}_{8}$ and $\mathrm{C}_{11}$ (Kubo et al., 2004). Also, a similar MIC value for the octyl gallate $(25 \mu \mathrm{g} / \mathrm{mL})$ has been reported against 2 Staph. aureus strains (Kubo et al., 2003).

Combinations of antimicrobial compounds are more effective than single preservatives because of their multiple modes of action. In this study, we used binary combinations of phenolic compounds, and the amount of each required to achieve growth inhibition was reduced from about 2 to 8 times, depending on the combinations with regard to the individual values. For BHA and octyl gallate (synthetic compounds), the MIC were reduced 4 times in the presence of gallic acid (natural phenolic compound) against all strains tested. Importantly, no antagonism between natural + natural and natural + synthetic phenolic compounds was observed. On the other hand, we found synergistic effects in the combination of thymol + carvacrol and octyl gallate + gallic acid against the 4 E. faecalis strains of dairy origin. Interestingly, we also found partial synergy in the combinations gallic acid + thymol and octyl gallate + thymol against strains 189 and 297, which are resistant to 2 antibiotics, respectively. To our knowledge, no previous studies have been published concerning the combined effect of phenolic compounds against E. faecalis strains, although some data are available on the effect of combinations of particular phenolic compounds (flavonoids) and one antibiotic. Hence, Liu et al. (2001) found that $67 \%$ of clinically isolated vancomycin-resistant enterococci strains demonstrated a noticeable reduction in their resistance when vancomycin was combined with galangin $(12.5 \mathrm{mg} / \mathrm{mL})$ or 3,7-dihydroxyflavone $(6.25 \mathrm{mg} / \mathrm{mL})$, whereas no flavonoid alone inhibited these strains at $250 \mathrm{mg} / \mathrm{mL}$.

The synergism observed between thymol and carvacrol against the 4 strains of E. faecalis tested in this study, but not when these compounds were combined with BHA and octyl gallate (additive and partial synergy), might be attributed to their combined targeting of the cytoplasmic membrane, because they act on this structure as their primary target. Thymol and carvacrol are known to be inhibitors of foodborne microorganisms, although a drawback with the use of these compounds in food is their strong taste. This may not be a problem within a synergistic combination (such as most of the combinations with thymol or carvacrol described in this study), as one or both compounds in the combination could be used at a relatively low concentration. Synergism has also been reported for combinations of preservatives that separately have different mechanisms or sites of action on the microbial cell (Hugbo, 1977; Denyer et al., 1985). This could explain the synergism observed in this study between gallic acid and its alkyl derivative, octyl gallate. The latter compound is more hydrophobic and it could disrupt the cytoplasmic membrane, thus permitting easier access of gallic acid to the cell interior.

\section{CONCLUSIONS}

This study showed the antimicrobial effect of 3 natural and 2 synthetic phenolic compounds, tested alone or in binary combination under a standardized microdilution assay, against 4 dairy isolates of E. faecalis with different virulence factors. A general overview of the results indicated few differences among the different strains studied. Taking into account the MIC data of these 5 phenolic compounds in combination and the maximum usage levels commonly permitted in foods (200 mg/ $\mathrm{kg}$ based on the fat or oil content of the food product, equivalent to $200 \mu \mathrm{g} / \mathrm{mL}$ ) according to European Union legislation, carvacrol in combination with thymol (for all strains) or with BHA or octyl gallate (for 2 strains), as well as this synthetic compound (octyl gallate) in combination with carvacrol or gallic acid (for all strains) would be effective for the control of $E$. faecalis in milk or dairy products.

\section{ACKNOWLEDGMENTS}

This study was financially supported by La Junta de Castilla y León (Valladolid, Spain; JCyL ref. LE012A10).

\section{REFERENCES}

Barry, A. L. 1976. The antimicrobic susceptibility test: Principles and practices. Lea and Febiger, Philadelphia, PA.

Ben Arfa, A., S. Combes, L. Preziosi-Belloy, N. Gontard, and P. Chalier. 2006. Antimicrobial activity of carvacrol related to its chemical structure. Lett. Appl. Microbiol. 43:149-154.

Davidson, P. M., and M. E. Parish. 1989. Methods for testing the efficacy of food antimicrobials. Food Technol. 43:148-155.

Davidson, P. M., and T. M. Taylor. 2007. Chemical preservatives and natural antimicrobial compounds. Pages 713-745 in Food Microbiology: Fundamentals and Frontiers. 3rd ed. P. M. Doyle and I. R. Beuchat, ed. ASM Press, Washington, DC.

Denyer, S. P., W. B. Hugo, and V. D. Hardling. 1985. Synergy in preservative combinations. Int. J. Pharm. 25:245-253. 
EAFUS (Everything Added to Food In the United States). 2011. EAFUS database. Accessed Nov. 12, 2012. http://www.fda.gov/Food/ IngredientsPackagingLabeling/ucm115326.htm.

EFSA (European Food Safety Authority). 2008. Report from the Task Force on Zoonoses Data Collection including guidance for harmonized monitoring and reporting of antimicrobial resistance in commensal Escherichia coli and Enterococcus spp. from food animals. EFSA J. 141:1-44.

EFSA (European Food Safety Authority). 2011. Scientific opinion on the maintenance of the list of QPS biological agents intentionally added to food and feed (2011 update). EFSA J. 9:2497.

Gailani, M. B., and D. Y. C. Fung. 1984. Antimicrobial effects of selected antioxidants in laboratory media and in ground pork. J. Food Prot. 47:428-433.

Garrote, G., J. M. Cruz, A. Moure, H. Domínguez, and J. C. Parajó. 2004. Antioxidant activity of byproducts from the hydrolytic processing of selected lignocellulosic materials. Trends Food Sci. Technol. 15:191-200

Griffiths, M. W. 2010. Bacillus cereus and other Bacillus spp. Pages 1-19 in Pathogens and Toxins in Foods. Challenges and Interventions, V. K. Juneja and J. Sofos, ed. ASM Press, Washington, DC.

Gutiérrez-Larraínzar, M., J. Rúa, I. Caro, C. de Castro, D. de Arriaga, M. R. García-Armesto, and P. del Valle. 2012. Evaluation of antimicrobial and antioxidant activities of natural phenolic compounds against foodborne pathogens and spoilage bacteria. Food Contr. 26:555-563.

Gutiérrez-Larraínzar, M., J. Rúa, D. de Arriaga, P. del Valle, and M. R. García-Armesto. 2013. In vitro assessment of synthetic phenolic antioxidants for inhibition of foodborne Staphylococcus aureus, Bacillus cereus and Pseudomonas fluorescens. Food Contr. 30:393-399.

Horrevorts, A. M., C. M. de Ridder, M. C. Poot, M. J. A. de Jonge, J. E. Degener, G. Dzoljic-Danilovic, M. F. Michel, and K. F. Kerrebijn. 1987. Checkerboard titrations: the influence of the composition of serial dilutions of antibiotics on the fractional inhibitory concentration index and fractional bactericidal concentration index. J. Antimicrob. Chemother. 19:119-125.

Hsieh, M. H., C. M. Yu, V. L. Yu, and J. W. Chow. 1993. Synergy assessed by checkerboard. A critical analysis. Diagn. Microbiol. Infect. Dis. 16:343-349.

Hugbo, P. G. 1977. Additivity and synergism in vitro as displayed by mixtures of some commonly employed antibacterial preservatives. Cosmetic Toiletries 92:52-56.

Hyldgaard, M., T. Mygind, and R.L. Meyer., 2012. Essential oils in food preservation: Mode of action, synergies, and interactions with food matrix components. Front. Microbiol. 3:1-24. http://dx.doi. org/10.3389/fmicb.2012.00012.

ICMSF (International Commission on Microbiological Specifications for Foods). 1978. Microorganisms in Foods 1: Their Significance and Methods of Enumeration. 2nd ed. University of Toronto Press, Toronto, Canada.

ISO (International Organization for Standardization). 2006. Clinical laboratory testing and in vitro diagnostic test systems-Susceptibility testing of infectious agents and evaluation of performance of antimicrobial susceptibility test devices-Part 1: Reference method for testing the in vitro activity of antimicrobial agents against rapidly growing aerobic bacteria involved in infectious diseases. ISO 20776-1: 2006. ISO, Geneva, Switzerland.

Jamet, E., E. Akary, M.-A. Poisson, J.-F. Chamba, X. Bertrand, and P. Serror. 2012. Prevalence and characterization of antibiotic resistant Enterococcus faecalis in French cheeses. Food Microbiol. 31:191-198.

Kubo, I., K. Fujita, K. Nihei, and N. Masuoka. 2003. Non-antibiotic antibacterial activity of dodecyl gallate. Bioorg. Med. Chem. 11:573-580.

Kubo, I., K.-L. Fujita, K. Nihei, and A. Nihei. 2004. Antibacterial activity of alkyl gallates against Bacillus subtilis. J. Agric. Food Chem. 52:1072-1076.

Liu, L. X., D. G. Durham, and M. E. Richards. 2001. Vancomycin resistance reversal in enterococci by flavonoids. J. Pharm. Pharmacol. 53:129-132.
López-Malo, A., E. Palou, and S. M. Alzamora. 2005. Naturally occurring compounds: Plant sources. Pages 429-452 in Antimicrobials in Food. 3rd ed. P. M. Davidson, J. N. Sofos and A. N. Branen, ed. CRC Press, Taylor and Francis Group, Boca Raton, FL.

Malani, P. N., C. A. Kauffman, and M. J. Zervos. 2002. Enterococci disease, epidemiology and treatment. Pages 305-408 in The Enterococci: Pathogenesis, Molecular Biology and Antibiotic Resistance. M. S. Gilmore, D. B. Clewell, P. Courvalin, G. M. Dunny, B. E. Murray and L. B. Rice, ed. ASM Press, Washington, DC.

Martins, L., M. G. Siqueira, P. Lynn, and R. Fackland. 2011. Enterococcus. Pages 350-364 in Manual of Clinical Microbiology. J. Versalovic, ed. ASM Press, Washington, DC.

Monteagudo-Mera, A., I. Caro, L. B. Rodríguez-Aparicio, J. Rúa, M. A. Ferrero, and M. R. García-Armesto. 2011. Characterization of certain bacterial strains for potential use as starter or probiotic cultures in dairy products. J. Food Prot. 74:1379-1386.

Monteagudo-Mera, A., L. Rodríguez-Aparicio, J. Rúa, H. MartínezBlanco, N. Navasa, M. R. García-Armesto, and M. A. Ferrero. 2012. In vitro evaluation of physiological probiotic properties of different lactic acid bacteria strains of dairy and human origin. J. Funct. Foods 4:531-541.

Ogier, J.-C., and P. Serror. 2008. Safety assessment of dairy microorganisms: The Enterococcus genus. Int. J. Food Microbiol. 126:291-301.

Pillar, C. M., and S. Gilmore. 2004. Enterococcal virulence-pathogenicity island of E. faecalis. Front. Biosci. 9:2335-2346.

R Development Core Team. 2009. R: A language and environment for statistical computing. R Foundation for Statistical Computing, Vienna, Austria. http://www.R-project.org.

Ramos-Nino, M. E., M. N. Clifford, and M. R. Adams. 1996. Quantitative structure activity relationship for the effect of benzoic acids, cinnamic acids and benzaldehydes on Listeria monocytogenes. J. Appl. Bacteriol. 80:303-310.

Rúa, J., L. Fernández-Álvarez, C. de Castro, P. del Valle, D. de Arriaga, and M. R. García-Armesto. 2011. Antibacterial activity against foodborne Staphylococcus aureus and antioxidant capacity of various pure phenolic compounds. Foodborne Pathog. Dis. 8:149-157.

Rúa, J., L. Fernández-Álvarez, M. Gutiérrez-Larraínzar, P. del Valle, D. de Arriaga, and M. R. García-Armesto. 2010. Screening of phenolic antioxidants for their inhibitory activity against foodborne Staphylococcus aureus strains. Foodborne Pathog. Dis. 7:695-705.

Sánchez-Maldonado, A. F., A. Schieber, and M. G. Gänzle. 2011. Structure-function relationships of the antibacterial activity of phenolic acids and their metabolism by lactic acid bacteria. J. Appl. Microbiol. 111:1176-1184.

Sato, M., H. Tanaka, R. Yamaguchi, K. Kato, and H. Etoh. 2004 Synergistic effects of mupirocin and an isoflavanone isolated from Erythrina variegata on growth and recovery of methicillin-resistant Staphylococcus aureus. Int. J. Antimicrob. Agents 24:241-246.

Shahidi, F., and Ch.-T. Ho. 2005. Phenolics in food and natural health products: An overview. Pages 1-8 in Phenolic Compounds in Foods and Natural Health Products. F. Shahidi and C.-T. Ho, ed. ACS Symposium Series 909, American Chemical Society, Washington, DC.

Shankar, N., A. S. Baghdavan, and M. S. Gilmore. 2002. Modulation of virulence within a pathogenicity island in vancomycin resistant Enterococcus faecalis. Nature 417:746-750.

Sikkema, J., J. A. M. De Bont, and B. Poolman. 1995. Mechanism of membrane toxicity of hydrocarbons. Microbiol. Rev. 59:201-222.

Svensson, L., B. Sekwati-Monang, D. Lopes Lutz, A. Schieber, and M. G. Gänzle. 2010. Phenolic acids and flavonoids in nonfermented and fermented red sorghum (Sorgum bicolor (L.) Moench). J. Agric. Food Chem. 58:9214-9220.

Tendolkar, P. M., A. S. Baghdayan, and N. Shankar. 2003. Pathogenic enterococci new developments in the 21st century. Cell. Mol. Life Sci. 60:2622-2636.

Ultee, A., M. H. J. Bennik, and R. Moezelaar. 2002. The phenolic hydroxyl group of carvacrol is essential for action against the foodborne pathogen Bacillus cereus. Appl. Environ. Microbiol. $68: 1561-1568$. 